\title{
Preference for instrumental and non-instrumental information
}

ROBERT J. WOLOSIN AND MYRNA A. WOLOSIN

THE UNIVERSITY OF MICHIGAN

Recent studies have indicated that humans seek information about unavoidable but uncertain outcomes. This response has been interpreted according to a preparatory-state hypothesis which maintains that the information prepares the $S$ emotionally for the outcome. The current study seeks to ascertain whether information about an already known outcome will be sought. Half the Ss could request information about a to-be-delivered outcome; the other half could request information about an outcome which had already been delivered. Ss in the latter condition requested information more than in the former, and this unexpected effect raises some question as to the validity of the preparatory-state hypothesis.

In a recent study, Lanzetta \& Driscoll (1966) have shown that humans seek information about uncertain outcomes even though they can do nothing to change them. Using electric shock and monetary reward as outcomes, they found that Ss preferred to know about these outcomes in advance, although they could not use the information to affect the outcome in any way. Among others, Lanzetta and Driscoll suggested that Ss use the information in order to prepare themselves emotionally for a positive or negative outcome. However, galvanic skin response (GSR) data supported the preparatory-state hypothesis only in part. Lanzetta and Driscoll found differential GSRs to shock and reward when the Ss recelved information prior to these outcomes, and similar GSRs without information. But this effect was limited to one of three experimental conditions.

In order to test the preparatory-response hypothesis further, the present study utilized a situation in which the information had no preparatory value. When information cannot influence outcomes, it can have preparatory emotional value only if outcomes are uncertain. The question arises, then, if individuals will seek information about unavoidable outcomes that are fully certain, or have already taken place. Method

Ss were 20 undergraduate males, some from the University of Michigan paid-subject pool, and some recruited from introductory psychology classes at the University.

The $S$ sat at a table in an observation room equipped with one-way mirrors. In front of him was a screen onto which slides were projected from outside the room. The $S$ had two buttons, one in each hand, to indicate his responses. A buzzer was used as the warning signal for the beginning of a trial. Outside the room was a slide projector, a device for initiating the warning signal, and an Esterline-Angus Event Recorder. One $E$ was always outside the room, initiating each trial and projecting slides; the other read the instructions to the $S$ and administered rewards and punishments-he was always present in the experimental room, sitting to the left of the $S$.

Each $S$ was given 30 trials on which he had the choice of acquiring or not acquiring information about which of two equally likely outcomes would occur or had already occurred. There were three blocks of 10 trials, each presenting a different combination of outcomes. The three combinations were: win-tie; losetie; and win-lose. The third combination was always presented last, and the first two were counterbalanced for order. Ss were randomly assigned to one of two conditions. Both conditions employed the Lanzetta and Driscoll paradigm, with the following exceptions: (1) In place of a token reward, which could later be exchanged for money (each token worth 10 cents), dimes were used. (2) In place of shock, dimes were taken from the S. (3) Ten trials, rather than 30 , were run for each outcome combination. (4) In the case of a non-information seeking response, $S$ was presented a stimulus (a standard slide-see below). (5) GSRs were not obtained, nor were response latencies.

The Ss in each condition were read a set of instructions explaining that $\mathrm{E}$ was interested in studying how individuals respond to pleasant and unpleasant outcomes. Ss were told that they could win a dime, lose a dime, or tie on each of a series of trials. These outcomes were based on a "match" between two pairs of digits. The digits were zeroes and ones. S's digits were on the left half of a slide, and E's were on the right half. S scored a "win" when the sum of his digits was larger than the sum of E's. A "loss" was the converse, and in a tie the sums were equal. There were five ways each to win, lose, and tie. Within a series each relevant way was used once in a random order. It was emphasized that the outcomes were equally likely, and the $S$ could do nothing to alter them. In Condition 1 , the $S$ was told the monetary outcome of the trial before the onset of the buzzer, and a dime was either given to him, taken from him, or not exchanged. Then during the 5 sec warning buzzer, $S$ made either an information seeking response or a non-information seeking response. In the event of an information seeking response, he was shown 
for 5 sec the slide which gave him the win, loss, or tie. In the event of a non-information seeking response, he was shown, also for $5 \mathrm{sec}$, a standard slide containing four ones. There was a $5 \mathrm{sec}$ intertrial interval.

In Condition 2, the $S$ did not know the outcome before the buzzer came on. On each trial, the buzzer came on for $5 \mathrm{sec}$, and during this interval $\mathrm{S}$ could make either an information seeking response or a non-information seeking response. If an information seeking response was made, he was shown for $5 \mathrm{sec}$ a slide which gave him a win, loss, or tie. If a noninformation seeking response was made, he was shown the standard slide for $5 \mathrm{sec}$. Then the monetary outcome was delivered; a dime was either given to him, taken from him, or not exchanged. Again there was a 5 sec intertrial interval. Thus, for both conditions, each trial took $20 \mathrm{sec}$.

Subjects were given 10 dimes to begin with, and were told that the money they had left at the end would be theirs. Before each set of trials, E announced which outcomes would be possible and emphasized that they were equally likely.

\section{Results and Discussion}

In both conditions, the mean number of information seeking responses is significantly greater than chance for all cells except one (see Table 1). This was determined by testing each cell mean against the null hypothesis of five information seeking responses. A two way analysis of variance showed no significant effect due to outcome conditions, a significant conditions effect $(F=4.76, d f=1 / 54, p<.05)$, and a significant interaction $(F=20.07, \mathrm{df}=2 / 54, \mathrm{p}<.01)$. The percentages of information seeking responses in Condition 2 are quite similar to those found by Lanzetta and Driscoll (see Table 1).

Table 1.

Mean Percent of Information Seeking Responses over 10 Trials

\begin{tabular}{lccc} 
Treatment & \multicolumn{3}{c}{ Outcome combination } \\
& Win-tie & Lose-tie & Win-lose \\
\hline Condition 1 & 70.0 & 62.0 & 87.0 \\
Condition 2 & 66.0 & 62.0 & 62.0 \\
$\begin{array}{l}\text { Lanzetta \& Drisco!l } \\
\text { (1966; adjusted means) }\end{array}$ & 60.0 & 65.0 & 63.0 \\
\hline
\end{tabular}

\section{Errata}

Gold, D. The effect of generalized and specific expectancies upon eyelid conditioning. Psychon. Sci., $1967,8,147-148-$ The last sentence of the first paragraph on page 148 should read: "All eyelid closures occurring between 300 and $440 \mathrm{msec}$ were considered CRs."
Table 2. Frequency of Information Seeking Responses in Condition 1 as a Function of Announced Outcome and Outcome Combination

\begin{tabular}{lccc} 
Announced Outcome & \multicolumn{3}{c}{ Outcome combination } \\
& Win-tie & Lose-tie & Win-lose \\
\hline Win & 42 & -- & 46 \\
Lose & - & 37 & 41 \\
Tie & 28 & 25 & -- \\
\hline
\end{tabular}

When information seeking responses from Condition 1 are plotted as a function of the monetary outcome announced by $\mathrm{E}$ (Table 2), there are clear differences among the frequencies. These show that Ss in Condition 1 requested information most often when E announced a "win," and slightly less often when E announced a "loss." Ss did not seek information above the chance level after an announced "tie." An overall F-test showed these differences to be significant at the .01 level.

The fact that there was significantly greater preference for information about certain rather than uncertain outcomes casts doubt on the validity of the preparatory-response explanation for information seeking. The reason for this is as follows: The Lanzetta \& Driscoll (1966) findings, as well as the data from Condition 2, could be attributed to a preparatoryresponse mechanism, but the data from Condition 1 clearly cannot, since the outcome is already known to the S. It is possible that the preparatory-response hypothesis is true for that class of situations in which there is outcome uncertainty, and there is some other explanation which holds when there is no outcome uncertainty. It seems more economical, however, from the point of view of theory, to maintain that the underlying motive for the acquisition of information is the same in either case, and is yet to be found.

\section{Reference}

Lanzetta, J. T., \& Driscoll, J. M. Preference for information about an uncertain but unavoidable outcome. J. Pers. soc. Psychol., $1966,3,96-102$.

\section{Note}

1. This research was carried out at the Group Dynamics Laboratory, Institute for Social Research, Ann Arbor, Michigan. The authors wish to thank Dr. Robert B. Zajonc for his helpful suggestions.
Eisenman, R. Complexity-simplicity: I. Preference for symmetry and rejection of complexity. Psychon. Sci., 1967, 8, 169-170.-Several words were omitted from the abstract. The last three lines should be revised to read: "findings. In Study 1 it was found that Ss preferred symmetrical shapes to a highly significant extent. In Study 2, when Ss were presented with symmetrical shapes, Ss rejected complexity to a highly significant extent, although they did not necessarily prefer the simplest shapes." 